\title{
Erratum to: Health-related quality of life and mental health in parents of children with hemolytic uremic syndrome
}

\author{
Kathrin Buder $^{1}$ • Helene Werner ${ }^{2}$ - Markus A. Landolt ${ }^{2,3}$ - Thomas J. Neuhaus ${ }^{4}$. \\ Guido F. Laube ${ }^{1} \cdot$ Giuseppina Spartà $^{1}$
}

Published online: 5 April 2016

(C) IPNA 2016

\section{Erratum to: Pediatr Nephrol}

DOI 10.1007/s00467-015-3294-0

In the legends of Table 2 (footnote c), Table 4 (footnote $c$ ), and Table 5 (footnote $b$ ), the term "Cronbach's $\alpha$ " was wrongly mentioned. Instead, the indicated values refer to the effect size according to Cohen's $d$. Also, in all three of these tables a large effect is indicated by a Cohen's $d>0.80$ rather than $>80$.
In the legends of Table 2 and 4 (footnotes $d$ and $e$ in each case), the term "vs." was mistakenly used instead of the correct "minus".

In Table 4, the wrong superscript - indicating which statistical test was applied - was used in two places. Comparison of maternal and paternal mental health scores with normative data was tested by using one-sample $t$ tests; the comparison of mothers' and fathers' mental health scores was tested by using Wilcoxon signed-rank tests.

The correct versions of Tables 2, 4 and 5 are shown here.

The online version of the original article can be found at http://dx.doi.org/ 10.1007/s00467-015-3294-0.

Giuseppina Spartà

giuseppina.sparta@kispi.uzh.ch

1 Pediatric Nephrology Unit, University Children's Hospital Zurich, Steinwiesstrasse 75, 8032 Zurich, Switzerland

2 Department of Psychosomatics and Psychiatry, University Children's Hospital Zurich, Steinwiesstrasse 75, 8032 Zurich, Switzerland

3 Department of Child and Adolescent Health Psychology, Institute of Psychology, University of Zurich, Binzmuehlestrasse 14, 8051 Zurich, Switzerland

4 Children's Hospital of Lucerne, Cantonal Hospital of Lucerne, 6000 Lucerne 16, Switzerland 
Table 2 Health-related quality of life of mothers and fathers with HUS-affected children, compared with population norm and between mothers and fathers

\begin{tabular}{|c|c|c|c|c|c|c|c|c|}
\hline \multirow[t]{2}{*}{ SF-36 scales } & \multirow[t]{2}{*}{ Mothers $(n=61-63)$} & \multicolumn{2}{|c|}{$\begin{array}{l}\text { Comparison of mothers' } \\
\text { HRQoL with female } \\
\text { references (normative data) }\end{array}$} & \multirow[t]{2}{*}{ Fathers $(n=58)$} & \multicolumn{2}{|c|}{$\begin{array}{l}\text { Comparison of fathers' } \\
\text { HRQoL with male } \\
\text { references (normative data) }\end{array}$} & \multicolumn{2}{|c|}{$\begin{array}{l}\text { Comparison of mothers' } \\
\text { and fathers' HRQoL }{ }^{\mathrm{b}} \\
(n=56-58)\end{array}$} \\
\hline & & $p$ & $d^{\mathrm{c}, \mathrm{d}}$ & & $p$ & $d^{c, \mathrm{~d}}$ & $p$ & $d^{\mathrm{c}, \mathrm{e}}$ \\
\hline Physical functions & $94.4 \pm 16.5$ & 0.02 & 0.30 & $96.2 \pm 8.0$ & $<0.001$ & 0.44 & 0.88 & -0.14 \\
\hline Role physical & $91.1 \pm 20.9$ & 0.01 & 0.27 & $97.4 \pm 10.1$ & $<0.001$ & 0.46 & 0.04 & -0.38 \\
\hline Bodily pain & $83.8 \pm 22.0$ & $<0.001$ & 0.77 & $90.0 \pm 16.6$ & $<0.001$ & 0.88 & 0.09 & -0.32 \\
\hline General health & $80.8 \pm 16.1$ & $<0.001$ & 0.69 & $77.6 \pm 16.1$ & $<0.001$ & 0.59 & 0.30 & 0.20 \\
\hline Vitality & $65.7 \pm 17.1$ & $<0.001$ & 0.48 & $67.1 \pm 17.2$ & 0.20 & 0.17 & 0.73 & -0.08 \\
\hline Social functions & $89.3 \pm 18.5$ & 0.05 & 0.24 & $90.7 \pm 15.6$ & 0.46 & 0.09 & 0.49 & -0.08 \\
\hline Role emotional & $94.5 \pm 20.4$ & $<0.01$ & 0.30 & $96.0 \pm 15.4$ & 0.05 & 0.20 & 0.52 & -0.08 \\
\hline Mental health & $77.4 \pm 14.9$ & $<0.001$ & 0.47 & $76.7 \pm 15.9$ & 0.48 & 0.10 & 0.82 & 0.04 \\
\hline PCS & $54.3 \pm 7.6$ & $<0.001$ & 0.70 & $55.4 \pm 4.7$ & $<0.001$ & 0.81 & 0.70 & -0.18 \\
\hline MCS & $52.1 \pm 8.8$ & $<0.01$ & 0.34 & $51.6 \pm 8.2$ & 0.85 & -0.02 & 0.78 & 0.06 \\
\hline
\end{tabular}

Data on health-related quality of life (HRQoL) are presented as the mean \pm SD. The higher the value, the better the HRQoL

SF-36, Medical Outcomes Study Short Form-36 item questionnaire; PCS physical component summary score of SF-36; MCS, mental component summary score of SF-36

${ }^{\text {a }}$ Values are presented as the mean $\pm \mathrm{SD}$. One-sample $t$ tests were performed

${ }^{\mathrm{b}}$ Wilcoxon signed-rank tests were performed

${ }^{\mathrm{c}} d=$ Effect size according to Cohen [38], with a Cohen's $d$ value of 0.20 indicating a small effect; a $d$ value of 0.50 indicating amedium-sized effect; a $d$ value of $<0.80$ indicating a large effect)

${ }^{d}$ Differences: Study sample minus normative data (positive $d$ indicates better HRQoL for the study sample compared to the population norm)

e Differences: Mothers' minus fathers' data (positive d indicates better HRQoL for the mothers than for the fathers)

Table 4 Mental health of mothers and fathers with HUS-affected children, compared with population norm and between mothers and fathers

\begin{tabular}{|c|c|c|c|c|c|c|c|c|}
\hline \multirow[t]{2}{*}{ Brief Symptom Inventory scales } & \multirow[t]{2}{*}{ Mothers $(n=63)$} & \multicolumn{2}{|c|}{$\begin{array}{l}\text { Comparison of mothers' } \\
\text { mental health scores with } \\
\text { normative data }^{\mathrm{a}}\end{array}$} & \multirow[t]{2}{*}{ Fathers $(n=58)$} & \multicolumn{2}{|c|}{$\begin{array}{l}\text { Comparison of fathers' } \\
\text { mental health scores with } \\
\text { normative data }^{\mathrm{a}}\end{array}$} & \multicolumn{2}{|c|}{$\begin{array}{l}\text { Comparison of mothers' } \\
\text { and fathers' mental } \\
\text { health scores }{ }^{b}\end{array}$} \\
\hline & & $\bar{p}$ & $d^{c, d}$ & & $\bar{p}$ & $d^{\mathrm{c}, \mathrm{d}}$ & $p$ & $d^{\mathrm{c}, \mathrm{e}}$ \\
\hline Somatization & $47.8 \pm 8.9$ & 0.05 & -0.23 & $48.0 \pm 9.3$ & 0.10 & -0.21 & 0.79 & -0.02 \\
\hline Obsessive-compulsive & $46.6 \pm 10.4$ & 0.01 & -0.33 & $46.9 \pm 10.4$ & 0.03 & -0.30 & 0.37 & -0.03 \\
\hline Interpersonal sensitivity & $46.7 \pm 10.8$ & 0.02 & -0.32 & $47.6 \pm 8.5$ & 0.04 & -0.26 & 0.43 & -0.09 \\
\hline Depression & $46.6 \pm 9.7$ & $<0.01$ & -0.35 & $49.3 \pm 8.9$ & 0.53 & -0.08 & 0.01 & -0.29 \\
\hline Anxiety & $47.4 \pm 10.0$ & 0.05 & -0.26 & $47.3 \pm 9.8$ & 0.04 & -0.27 & 0.68 & 0.02 \\
\hline Hostility & $49.3 \pm 10.7$ & 0.58 & -0.07 & $49.5 \pm 9.5$ & 0.70 & -0.05 & 0.87 & -0.03 \\
\hline Phobic anxiety & $47.4 \pm 7.4$ & $<0.01$ & -0.30 & $48.4 \pm 7.4$ & 0.11 & -0.18 & $<0.001$ & -0.14 \\
\hline Paranoid ideation & $48.1 \pm 9.4$ & 0.12 & -0.19 & $50.5 \pm 9.9$ & 0.71 & 0.05 & 0.06 & -0.24 \\
\hline Psychoticism & $50.8 \pm 10.2$ & 0.55 & 0.08 & $48.2 \pm 7.8$ & 0.08 & -0.20 & 0.50 & 0.29 \\
\hline Global Severity Index & $45.0 \pm 13.2$ & $<0.01$ & -0.43 & $45.0 \pm 13.5$ & $<0.01$ & -0.42 & 0.96 & -0.01 \\
\hline
\end{tabular}

Data on the mental health of the parents are presented as the mean \pm SD. The higher the value, the lower the mental health score

${ }^{\text {a }}$ One-sample $t$ tests were performed

${ }^{\mathrm{b}}$ Wilcoxon signed-rank tests were performed

${ }^{\mathrm{c}} d=$ Effect size according to Cohen [38], with a Cohen's $d$ value of 0.20 indicating a small effect; a $d$ value of 0.50 indicating a medium-sized effect; a $d$ value of $<0.80$ indicating a large effect)

${ }^{\mathrm{d}}$ Differences: study sample minus normative data (negative $d$ indicates better mental health for the study sample compared to the population norm)

'Differences: Mothers' minus fathers' data (negative $d$ indicates better mental health for the mothers than for the fathers) 
Table 5 Prevalence of post-traumatic stress symptoms and post-traumatic stress disorder (PTSD) according to DSM-IV in 63 mothers and 58 fathers experiencing their child's hemolytic uremic syndrome (HUS) as a traumatic event

\begin{tabular}{|c|c|c|c|c|}
\hline \multirow[t]{2}{*}{ Posttraumatic Diagnostic Scale items } & \multirow[t]{2}{*}{ Mothers } & \multirow[t]{2}{*}{ Fathers } & \multicolumn{2}{|c|}{ Comparison between mothers and fathers ${ }^{\mathrm{a}}$} \\
\hline & & & $p$ & $d^{b}$ \\
\hline $\begin{array}{l}\text { Parents stating their child's HUS as a traumatic event } \\
\text { Intrusion }\end{array}$ & \multicolumn{3}{|c|}{ Intrusion } & \\
\hline DSM-IV criterion met & $20(32 \%)$ & $10(17 \%)$ & & \\
\hline Number of symptoms & $2.1 \pm 1.5[0-5]$ & $1.2 \pm 1.4[0-4]$ & 0.02 & 0.65 \\
\hline \multicolumn{5}{|l|}{ Avoidance } \\
\hline DSM-IV criterion met & $1(2 \%)$ & $2(4)$ & & \\
\hline Number of symptoms & $0.9 \pm 1.4[0-6]$ & $0.6 \pm 1.3[0-5]$ & 0.02 & 0.24 \\
\hline \multicolumn{5}{|l|}{ Hyperarousal } \\
\hline DSM-IV criterion met & $7(11 \%)$ & $6(10 \%)$ & & \\
\hline Number of symptoms & $1.1 \pm 1.5[0-5]$ & $0.9 \pm 1.2[0-3]$ & 0.11 & 0.17 \\
\hline \multicolumn{5}{|l|}{ All 3 symptom clusters } \\
\hline DSM-IV criteria for full PTSD met & $0(0 \%)$ & $2(4)$ & & \\
\hline Criteria for partial PTSD met & $4(6 \%)$ & $1(2)$ & & \\
\hline Total symptom severity & $6.1 \pm 6.7[1-30]$ & $3.1 \pm 4.0[0-15]$ & $<0.01$ & 0.54 \\
\hline
\end{tabular}

Data are presented as the mean \pm SD with the range given in square brackets, or as a number with the percentage in parenthesis

PTSD, Post-traumatic stress disorder; DSM-IV, Diagnostic and Statistical Manual of Mental Disorders (version IV)

${ }^{a}$ Wilcoxon signed-rank tests for continuous variables were performed

${ }^{\mathrm{b}} d=$ Effect size according to Cohen [38], with a Cohen's $d$ value of 0.20 indicating a small effect; a $d$ value of 0.50 indicating a medium-sized effect; a $d$ value of $<0.80$ indicating a large effect). Mothers' vs. fathers' data, with a positive $d$ indicating a higher number of PTSD symptoms for the mothers than for the fathers 